\title{
On Multiuser Switched Diversity Transmission for Spectrum Sharing Systems
}

\author{
Marwa Qaraqe ${ }^{\dagger}$, Mohamed Abdallah*, Erchin Serpedin ${ }^{\dagger}$ and Mohamed-Slim Alouini ${ }^{\ddagger}$ \\ ${ }^{\dagger}$ Department of Electrical and Computer Engineering, Texas A\&M University, College Station, TX, 77843-3128, USA \\ * Department of Electrical and Computer Engineering, Texas A\&M University at Qatar, Education City, 23874, Doha, Qatar \\ ${ }^{\ddagger}$ Electrical Engineering Program, KAUST, Thuwal, Makkah Province, Saudia Arabia \\ E-mails: marwa@tamu.edu,mohamed.abdallah@qatar.tamu.edu, serpedin@ece.tamu.edu,mohamed.alouini@kaust.edu.sa
}

\begin{abstract}
In this paper, we develop multiuser access schemes for spectrum sharing systems whereby secondary users share the spectrum with primary users. In particular, we devise two schemes for selecting the user among those that satisfy the interference constraints and achieve an acceptable signal-to-noise ratio (SNR) level. The first scheme selects the user with the maximum SNR at the receiver, whereas in the second scheme the users are scanned in a sequential manner until an acceptable user is found. In addition, we consider two power adaptive settings. In the on/off power adaptive setting, the users transmit based on whether the interference constraint is met or not while in the full power adaptive setting, the users vary their transmission power to satisfy the interference constraint. Finally, we present numerical results of our proposed algorithms where we show the trade-off between the average spectral efficiency and average feedback load of both schemes.
\end{abstract}

Index Terms-Multiuser Diversity, Switched Diversity, Spectrum Sharing, Performance Analysis

\section{INTRODUCTION}

Recent studies conducted by the United States Federal Communications Commission (FCC) indicate that the wireless spectrum is largely underutilized, and the temporal and geographical variation in the utilization of the assigned spectrum ranges from $15 \%$ to $85 \%$ [1]. The fixed spectrum assignment policy served well in the past; however, the recent need for anytime-anywhere service access over the wireless network has created an urgent demand for wireless radio resources [2]. This demand, as well as the underutilization of the spectrum, gave the concept of cognitive radio greater importance. First introduced by Mitola and Maguire [3], the basic idea of a cognitive radio is to enable the primary (licensed) user (PU) and secondary (unlicensed) user (SU) to coexist in the same frequency spectrum.

The cognitive radio technology is the key technology that enables NeXt Generation Networks to use the spectrum in a dynamic manner [3]. Dynamic Spectrum Access Networks (DSANs) facilitates cognitive radio (CR) devices to analyze the spectrum bands and access them if unoccupied until the incumbent transmitter arrives [2]. In underlay cognitive radio systems, also known as spectrum sharing systems, primary and secondary users can simultaneously transmit information as long as the interference of the secondary user to the primary user stays below a predetermined threshold, called the interference constraint [3].
In a multiuser communication system, multiuser diversity (independent fading channels across different users) can be exploited to maximize the average throughput [4]. In multiuser communication systems, a base station (BS) is connected to several users that are competing for channel access in a time division multiplexed (TDM) access mode. In general, the BS probes all users and selects the user that reports the best channel quality based on the channel state information (CSI) that is available through a feedback channel between the users and the BS. In a non-cognitive setting, the BS switches between users depending on a predefined SNR switching threshold [4]. This threshold ensures a reliable secondary communication transmission. In general, if a user's SNR is below the SNR threshold, the BS switches and examines other users [5].

In this paper, a multiuser-switched diversity transmission scheme is adapted to an underlay cognitive setting. Two types of schemes in two different settings are discussed and analyzed. In the first setting, the system operates in an On/Off power adaptive setting. A traditional way of performing this task is that for all users that do not meet the interference constraint with the primary user, the BS does not consider them for transmission and therefore their transmit power must be set to zero. If a user meets the interference constraint as well as the SNR switching threshold, then it is able to transmit with its maximum transmit power, assuming the BS selects it for transmission. In the second setting, the system operates in a full power adaptive mode. Specifically, the users that do not meet the interference constraint with the primary receiver must decrease their transmit power level so that they meet the max interference threshold. In this setting, all users adapt their power settings so that they meet the interference constraint and therefore all users compete for channel access based on whether they are able to meet the switching threshold [6].

The first scheme is equivalent to the selection combining transmission (SCT) scheme in a spatial diversity system. In SCT, the BS serves the user with the strongest channel and that simultaneously meets an interference constraint with the primary and the switching threshold for optimal secondary communication. This scheme yields the best average spectral efficiency (ASE), but comes at the expense of a high feedback load, (defined as the number of estimated users probed before channel access), since the BS must first probe all users and 
then select the user with the highest signal-to-noise ratio (SNR) [7]. In an attempt to simplify the selection procedure and reduce the feedback load, the scan-and-wait transmission (SWT) scheme is analyzed. In this scheme, the BS executes a sequential search of the user and selects the first acceptable user that meets the interference and switching constraints instead of the best user [8].

The remainder of this paper is organized as follows. Section II presents the system model and the adaptive modulation that is used, as well as the ASE and BER analysis. Section III presents the proposed access schemes in both power settings, the mode of operation of these schemes, and the performance analysis of the proposed schemes. Section IV offers several numerical examples that illustrate the performance of the analyzed schemes.

\section{System Model And Adaptive Modulation}

\section{A. System and Channel Model}

In this section, the system and channel models considered in the paper are briefly outlined. A time division multiplexed (TDM) system is assumed where only one user is allowed to have channel access per time-slot for uplink transmission. A single time-slot is divided in two regions, a guard time and an information transmission time. During the guard time the BS probes the users to find a user that will be given access to the channel in the succeeding information transmission time. The guard time is assumed to be fixed and equal to the amount of time that is needed to probe all users. The time duration of a single time-slot is assumed to be roughly equal to the channel coherence time. Under the assumption of frequency flat-fading, we use a block-fading model, assuming that each data burst experiences the same fading conditions as the preceding guard period.

For simplicity, each individual user and the BS are equipped with a single antenna, and perfect channel state information (CSI) is assumed to be available at both the BS and the users. For our study we assume independent and identically distributed (i.i.d) Rayleigh fading channels across the different users.

We let $h_{p_{i}}$ be the channel coefficient between the $i^{\text {th }}$ user and the primary receiver (PR) and $h_{s_{i}}$ be the channel coefficient between the $i^{\text {th }}$ user and the BS. We assume that $h_{p}$ and $h_{s}$ are zero-mean complex Gaussian random variables with variances $\sigma_{p}^{2}$ and $\sigma_{p}^{2}$, respectively. We also let $\left|h_{p_{i}}\right|^{2}$ and $\left|h_{s_{i}}\right|^{2}$ denote the instantaneous channel gains from the $i^{t h}$ secondary user to the PR and from the $i^{t h}$ secondary user to the BS, respectively. Assuming a transmit power of $P_{t}$ and Gaussian noise with zero mean and variance $N_{o}$ on both the secondary and interference channels, the received interference SNR with the primary and the received secondary SNR at the $\mathrm{BS}$ are given respectively by

$$
\gamma_{p_{i}}=\frac{P_{t}\left|h_{p_{i}}\right|^{2}}{N_{o}}
$$

and

$$
\gamma_{s_{i}}=\frac{P_{t}\left|h_{s_{i}}\right|^{2}}{N_{o}}
$$

\section{B. Adaptive Transmission System}

Furthermore, we consider a constant-power, variable-rate, uncoded M-ary quadrature amplitude modulation (QAM) as the adaptive modulation system for our proposed schemes [7]. With this adaptive modulation, the SNR range is divided into $N+1$ fading regions and the constellation size $M=2^{n}$ (where $n=0,1, \ldots, N$ is the number of bits per symbol) is assigned to the $n$-th region. In depth, rate adaption is achieved by dividing the SNR range into $N+1$ regions which are defined by the constellation SNR thresholds $\left\{\gamma_{n}\right\}_{n=1}^{N}$. A data rate of $R_{n}=n$ is used if the reported received secondary SNR $\left(\gamma_{s_{i}}\right)$ of the chosen user satisfies the following inequality: $\gamma_{n} \leq \gamma_{s_{i}}<\gamma_{n+1}$. Note that the lower limit, $\gamma_{n}$, of each fading region is equal to lowest SNR which guarantees that the predefined target bit-error-rate (BER) is achieved by code $n$. The BER of a $2^{n-}$ QAM constellation with an SNR of $\gamma$ can be expressed as [9]:

$$
P_{b_{n}}(\gamma)=\frac{1}{5} \exp \left(\frac{-3 \gamma}{2\left(2^{n}-1\right)}\right)
$$

Given a target $\mathrm{BER}$ equal to $\mathrm{BER}_{o}$, the region boundaries for $\mathrm{n}=0,1, \ldots, \mathrm{N}$ are given by $[2, \mathrm{Eq}(4)]$

$$
\gamma_{n}=\frac{-2}{3} \ln \left(5 \mathrm{BER}_{0}\right)\left(2^{\mathrm{n}}-1\right)
$$

\section{ASE and BER Analysis}

The ASE of the systems in this paper is obtained as a sum of the spectral efficiencies $\left\{R_{n}\right\}_{n=1}^{N}=\{1,2, \ldots, N\}$ for the individual codes weighted by the probability $P_{n}$ that code $n$ is used:

$$
\mathrm{ASE}=\sum_{n=1}^{N} R_{n} P_{n}
$$

where

$$
P_{n}=\int_{\gamma_{n}}^{\gamma_{n+1}} f_{\gamma_{B S}}(x) d x=F_{\gamma_{B S}}\left(\gamma_{n+1}\right)-F_{\gamma_{B S}}\left(\gamma_{n}\right) .
$$

The functions $f_{\gamma_{B S}}(x)$ and $F_{\gamma_{B S}}$ denote the probability density function (PDF) and the cumulative distribution function (CDF) of the received SNR at the BS. The distribution of the PDF and CDF depends on the mode of operation of the selected multiuser access scheme. The BER, when averaged over all codes and SNRs, is given as the average number of erroneous bits divided by the average number of bits transmitted and is shown as follows:

$$
\overline{\mathrm{BER}}=\frac{\sum_{n=1}^{N} R_{n} \overline{\mathrm{BER}}_{n}}{\mathrm{ASE}}
$$

where $\overline{\mathrm{BER}}_{n}$ is the average BER for constellation size $n$ and is given by [2] [10]:

$$
\overline{\operatorname{BER}}_{n}=\int_{\gamma_{n}}^{\gamma_{n+1}} \operatorname{BER}(\mathrm{x}) \mathrm{f}_{\gamma_{\mathrm{BS}}}(\mathrm{x}) \mathrm{dx} .
$$




\section{Multiuser Access Schemes in Spectrum SHARING SYSTEMS}

In underlay cognitive systems, the secondary user is allowed to share the spectrum with the primary user as long as the secondary user's interference with the PR stays below an interference threshold, called the peak interference constraint (PIC) and denoted by Q. The PIC represents the maximum allowable interference power level that a primary user accepts. The second threshold a user must satisfy is the secondary switching threshold, $\gamma_{T}$. To ensure reliable secondary communication, the received SNR from a user at the BS must be at least $\gamma_{T}$. The switching thresholds in this paper are chosen to maximize the ASE (this can be seen in the analysis section of each scheme). Figure 1 depicts the system model where PT denotes the primary transmitter, PR denotes the primary receiver, and $U_{i}$ represents the $i^{t h}$ secondary user.

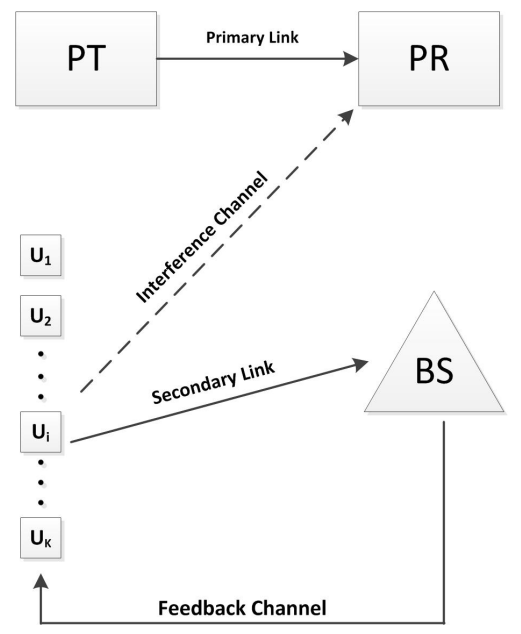

Fig. 1. System Model

\section{A. Selection Combining Access Scheme}

The selection combining transmission (SCT) scheme may be viewed as the optimal scheme when compared to the scanand-wait since it produces a higher ASE; however, SC comes with the disadvantage of a higher feedback load. Assuming that there are a total of $\mathrm{K}$ users connected to the BS, we examine the SC scheme as follows: during the guard time the BS probes all users and selects those users that meet the PIC with the primary $\left(\gamma_{p_{i}} \leq Q\right)$, thus receiving an acknowledgment from the PR, in an ACK/NACK signaling system. Next the BS probes the subset of K users that meet the PIC (known as the feedback user), asking them for their SNR and determines which user meets the switching threshold $\left(\gamma_{s_{i}} \geq \gamma_{T}\right)$. The BS communicates with these users through a direct feedback channel. Of the users that exceed the switching threshold, the BS chooses the user with the best channel quality (user with the largest $\gamma_{s}$ ). If all $\mathrm{K}$ users fail to meet the PIC or switching threshold, then the last user is picked (for simplicity), however, the selected user is not allowed to transmit anything in the subsequent transmission time. The
BS starts a new search after waiting a period longer than the channel coherence time.

\section{B. Scan-and-Wait Access Scheme}

In the scan-and-wait transmission scheme (SWT), the BS is no longer looking for the best user, but rather an acceptable user that meets both the PIC and the switching threshold. The SWT scheme works as follows: during the guard period, the BS initiates a sequential search of the users, requesting the $\operatorname{SNR}\left(\gamma_{s_{i}}\right)$ of each user while simultaneously comparing it with the ACK or NACK that is received from the PR (determining whether the user meets the PIC or not, respectively). If the $i^{t h}$ user receives an ACK from the PR and $\gamma_{s_{i}} \geq \gamma_{T}$, then that user is given access to the channel to either upload or download information during the following transmission period. If the $i^{t h}$ user fails to meet both or one of the conditions, the BS continues on and probes/checks the next user. This probing process continues until either a user is found that satisfies both constraints (this user is selected for the subsequent transmission time) or all $\mathrm{K}$ users fail to meet one or both constraints, in which case the BS simply picks the last user but does not allow it to transmit. Once again the BS waits a period longer than the channel coherence time before it starts a new sequential search. The BS begins its search from the user that was last given channel access.

\section{On/Off Power Adaptive Multiuser Access Schemes}

In this section, the performance of the SCT and SWT in the On/Off power adaptive mode is analyzed in terms of the ASE, average feedback load, and average delay. In the case of i.i.d Rayleigh fading channels across the different users, the received SNR from the $i^{\text {th }}$ user at the BS and at the PR, denoted by $\gamma_{s_{i}}$ and $\gamma_{p_{i}}$ respectively, are exponentially distributed for $i=1, \ldots, \mathrm{K}$. Their CDF and PDF are given by

$$
F_{\gamma_{s}}(x)=1-\exp \left(-\frac{x}{\bar{\gamma}_{s}}\right), x \geq 0
$$

and

$$
f_{\gamma_{s}}(x)=\frac{1}{\bar{\gamma}_{s}} \exp \left(-\frac{x}{\bar{\gamma}_{s}}\right), x \geq 0
$$

respectively, where $\bar{\gamma}_{s}$ is the common average faded SNR for the secondary link. The CDF and PDF of $\gamma_{p}$ are identical to (9) and (10) above but replacing $\bar{\gamma}_{s}$ with $\bar{\gamma}_{p}$ (the average faded SNR for the interference channel).

Analyzing the probability that no users is able to meet one or both constraints, the probability of no transmission of the SCT and SWT scheme is given by

$P_{n o}=\sum_{j=0}^{K}\left(\begin{array}{c}K \\ j\end{array}\right)\left(F_{\gamma_{p}}(Q)\right)^{j}\left[1-F_{\gamma_{p}}(Q)\right]^{K-j}\left(F_{\gamma_{s}}\left(\gamma_{T}\right)\right)^{j}$

1) Performance of the SCT Scheme: The operation of selecting a user but not transmitting anything in the case when all $\mathrm{K}$ users fail to be selected by the BS ensures that the probability of not exceeding $\gamma_{T}$ will be different from zero. Since no user is allowed to transmit information if all fail to be selected, the correct ASE and BER expressions are obtained 
by letting $\left\{R_{n}\right\}_{n=1}^{N}=0$ when $x<\gamma_{T}$. For simplicity, we let $\gamma_{s}^{*}$ denote the received SNR at the BS post scheduling. We compute the CDF of $\gamma_{s}^{*}$ per-time-slot for two cases. First, when $x<\gamma_{T}$, the $\mathrm{CDF}$ is equal to the probability that all users fail to be selected and the last selected user's SNR at the BS is below $x$, and is given by

$$
F_{\gamma_{s}^{*}}^{S C}(x)=P_{n o}
$$

where $F_{\gamma_{s}}(\cdot)$ is the CDF of the user's SNR at the BS given in (9). For the second case $\left(x \geq \gamma_{T}\right)$, the CDF is given by

$$
\begin{aligned}
F_{\gamma_{s}^{*}}^{S C}(x)= & \sum_{j=0}^{K}\left(\begin{array}{c}
K \\
j
\end{array}\right)\left(F_{\gamma_{p}}(Q)\right)^{j}\left[1-F_{\gamma_{p}}(Q)\right]^{K-j} \\
& \times \sum_{i=0}^{j}\left(\begin{array}{l}
j \\
i
\end{array}\right)\left(F_{\gamma_{s}}\left(\gamma_{T}\right)\right)^{j-i}\left[F_{\gamma_{s}}(x)-F_{\gamma_{s}}\left(\gamma_{T}\right)\right]^{i}
\end{aligned}
$$

Replacing $F_{\gamma_{B S}}(x)$ in (6) with $f_{\gamma_{s_{S C}}^{*}}(x)$, the ASE of the SC scheme is as follows

$$
\mathrm{ASE}_{\mathrm{SC}}=\left\{\begin{array}{l}
R_{q}\left[F_{\gamma_{s}^{*}}^{S C}\left(\gamma_{q+1}\right)-F_{\gamma_{s}^{*}}^{S C}\left(\gamma_{T}\right)\right] \\
+\sum_{n=q+1}^{N-1} R_{n}\left[F_{\gamma_{s}^{*}}^{S C}\left(\gamma_{n+1}\right)-F_{\gamma_{s}^{*}}^{S C}\left(\gamma_{n}\right)\right] \\
+R_{N}\left[1-F_{\gamma_{s}^{*}}^{S C}\left(\gamma_{N}\right)\right], q \in\{1,2, \ldots, N-1\} \\
R_{N}\left[1-F_{\gamma_{s}^{*}}^{S C}\left(\gamma_{T}\right)\right], \quad q=N
\end{array}\right.
$$

where the index $q \in\{1,2, \ldots, N\}$ denotes the fading regions in which $\gamma_{T}$ is placed. Defining $\gamma_{T}$ (for fixed $\mathrm{K}, \bar{\gamma}_{s}$ and $\bar{\gamma}_{p}$ ) as the switching threshold that maximized the ASE, it follows that

$$
\gamma_{T}=\underset{\gamma_{1 \leq \gamma \leq \gamma_{N}}}{\arg \max } \operatorname{ASE}_{S C}
$$

Defining the feedback load, $F_{B}$, as the number of users that the BS must probe to determine whether they meet the switching threshold, the feedback load per time-slot is given by

$$
F_{B}=\sum_{j=1}^{K} j\left[F_{\gamma_{I}}^{\star}(Q)\right]^{j}\left[1-F_{\gamma_{I}}^{\star}(Q)\right]^{K-j} .
$$

Thus the average feedback load of the SCT scheme is given by

$$
{\overline{F_{B}}}^{S C}=\frac{F_{B}}{1-P_{n o}} .
$$

If none of the users are able to satisfy both the switching threshold and the inference constraint, the system has to delay transmission until an acceptable user is found. If we let D be the number of time-slots that the system transmits nothing until a successful transmission, the average number of delays is given by

$$
\bar{D}=\frac{P_{n o}}{1-P_{n o}} .
$$

2) Performance of SWT Scheme: The SW scheme is based on a sequential search of an acceptable user. If we let $P_{F}$ denote the probability that a single user fails to be selected, then

$$
P_{F} S W=1-F_{\gamma_{p}}(Q)\left[1-F_{\gamma_{p}}(Q)\right] .
$$

The CDF of the output SNR for the SW scheme is obtained by analyzing the mode of operation and once again noting that if no acceptable user is found, the BS choses the last user; however, it does not allow it to transmit anything. Under these conditions, the following CDF is obtained

$$
F_{\gamma_{s}^{*}}^{S W}(x)=\left\{\begin{array}{l}
\frac{1-P_{n o}^{S W}}{1-P_{F}^{S W}} F_{\gamma_{p}}(Q)\left[F_{\gamma_{s}}(x)-F_{\gamma_{s}}\left(\gamma_{T}\right)\right] \\
+\left(P_{F}\right)^{K}, \quad x \geq \gamma_{T} \\
P_{n o}, \quad x<\gamma_{T}
\end{array}\right.
$$

The ASE and optimal switching threshold is found in a similar manner to (14) and (15) respectively, replacing $F_{\gamma_{s}^{*}}^{S C}\left(\gamma_{T}\right)$ with $F_{\gamma_{s}^{*}}^{S W}(x)$ and $\mathrm{ASE}_{S C}$ with $\mathrm{ASE}_{S W}$.

Defining the feedback load, $F_{B}$, as the number of user the BS probes before channel access, we can concluded that $F_{B}$ is geometrically distributed where $F_{B}$ can take the values $1,2, \ldots, \mathrm{K}$. Therefore, the average feedback load is given by

$$
{\overline{F_{B}}}^{S W}=\frac{1}{1-P_{F} S W}
$$

If the BS probes all the users and is unable to find an acceptable user for transmission, the BS does not allow transmission in the following transmission period. If we let $\mathrm{D}$ be the number of time-slots that the system delays transmission until a successful transmission, the average number of delays is then given by

$$
\begin{aligned}
\bar{D}^{S W} & =\sum_{m=0}^{+\infty} m P(D=m) \\
& =\sum_{m=0}^{+\infty} m P_{n o}{ }^{m}\left(1-P_{n o}\right) \\
& =\frac{P_{n o}}{1-P_{n o}}
\end{aligned}
$$

\section{Full Power Adaptive Multiuser Access Schemes}

In all spectrum sharing systems, all secondary users that want to transmit data to a target secondary receiver must first ensure that they stay below a maximum interference threshold that the primary receiver can tolerate, $\mathrm{Q}$. If a user is unable to meet the PIC, it is not considered for transmission. In this section, we propose and analyze a power adaptive scheme that adapts the secondary user's transmit power $P_{t_{i}}$ according to $\left|h_{p_{i}}\right|^{2}$ to satisfy the PIC at the primary receiver. That is, a secondary transmitter/user allocates its peak power $\left(P_{\max }\right)$ for transmission if the interference constraint is satisfied with the peak power. Otherwise, it adaptively adjusts its transmit power to the allowable level so that the interference observed at the PR is below the maximum interference level of Q. 
Correspondingly, the transmit power of the $i^{t h}$ secondary user is given by [11]

$$
P_{t_{i}}=\min \left(P_{\max }, \frac{Q N_{o}}{\left|h_{p_{i}}\right|^{2}}\right) .
$$

With this power adaptation technique, the $i^{t h}$ user's SNR at the BS, $\gamma_{s_{i}}^{P A}$, and its CDF are given, respectively, by [9][10]:

$$
\gamma_{s_{i}}^{P A}=\frac{P_{t_{i}}\left|h_{s_{i}}\right|^{2}}{N_{o}}=\frac{\min \left(P_{\max }, \frac{Q N_{o}}{\left|h_{p_{i}}\right|^{2}}\right)\left|h_{s_{i}}\right|^{2}}{N_{o}}
$$

and

$$
\begin{gathered}
F_{\gamma_{s_{i}}^{P A}}(x)=P\left(\gamma_{s_{i}}^{P A} \leq x\right) \\
=1-\left(1-\frac{1}{1+\frac{\sigma_{s}^{2}}{\sigma_{p}^{2}} \frac{Q}{x}} e^{-\frac{Q N_{o}}{\sigma_{p}^{2} P \max }}\right) e^{-\frac{x N_{o}}{\sigma_{s}^{2} P_{\max }}} .
\end{gathered}
$$

Due to the power adaption that the users perform, each user is able to satisfy the PIC and therefore the scheduling process simplifies to only checking that a user's received SNR at the BS exceeds the switching threshold. We define the probability of no transmission for the full power adaptive case as

$$
P_{n o}^{P A}=\left[F_{\gamma_{s_{i}}^{P A}}\left(\gamma_{T}\right)\right]^{K} .
$$

1) Performance of the Full Power Adaptive Selection Combining Scheme: In this section we discuss the mode of operation of the power-adaptive SC (SC-PA) scheme, characterize its received SNR post scheduling, and analyze its performance. Since the secondary users all adapt their power so that they are able to satisfy the PIC, the scheduling process becomes dependent only on $\gamma_{s_{i}}^{P A}$ and $\gamma_{T}$. In the SC-PA scheme, the BS starts by asking all the users for their SNR and compares them to $\gamma_{T}$. The BS then chooses the user with the maximum $\gamma_{s}^{P A}$ among all the users that exceed the switching threshold. If all users are unable to meet the switching threshold, the BS chooses the last user; however, it does not allow it to transmit any information in the subsequent transmission time.

The CDF of $\gamma_{s}^{P A^{*}}$, the output SNR per time-slot, is as follows

$$
F_{\gamma_{s}^{P A^{*}}}^{S C A}(x)=\left\{\begin{array}{l}
\sum_{j=0}^{K}\left(\begin{array}{c}
K \\
j
\end{array}\right)\left(F_{\gamma_{s}^{P A}}\left(\gamma_{T}\right)\right)^{K-j} \\
{\left[F_{\gamma_{s}^{P A}}(x)-F_{\gamma_{s}^{P A}}\left(\gamma_{T}\right)\right]^{j}, x \geq \gamma_{T}} \\
P_{n o}^{P A}(x), x<\gamma_{T}
\end{array}\right.
$$

The ASE and optimal switching threshold is found in a similar manner to (14) and (15) respectively, replacing $F_{\gamma_{s}^{*}}^{S C}(x)$ with $F_{\gamma_{s}^{*}}^{S C-P A}(x)$ and $\mathrm{ASE}_{S C}$ with $\mathrm{ASE}_{S C-P A}$. Since all K users meet the PIC, the BS must probe all users and therefore the feedback load for the SC-PA scheme per time-slot is deterministic and equal to $\mathrm{K}$, the total number of users connected to the BS. Evaluating the average feedback load over all the time slot, we conclude that

$$
{\overline{F_{B}}}^{S C-P A}=\frac{K}{1-P_{n o}^{P A}} .
$$

The average delay is given by

$$
\bar{D}^{S C-P A}=\frac{P_{n o}^{P A}}{1-P_{n o}^{P A}} .
$$

2) Performance of the Full Power Adaptive Scan and Wait Scheme: In this section we discuss the mode of operation of the power-adaptive SWT scheme, characterize its SNR post scheduling, as well as analyze its performance. Since the secondary users all adapt their power so that they are able to satisfy the PIC, the scheduling process becomes dependent only on $\gamma_{s_{i}}^{P A}$ and $\gamma_{T}$. In the SW scheme, the BS starts a sequential search asking the first user for its SNR. If the first user's SNR exceeds $\gamma_{T},\left(\gamma_{s_{i}}^{P A} \geq \gamma_{T}\right)$, then that user is given channel access to either upload or download information. If the first user has a SNR value less than $\gamma_{T}$, then the BS switches to the next user and checks its SNR with $\gamma_{T}$. This probing and checking continues until either a successful user is found and given channel access, or until all users fail to meet the switching threshold. In the latter case the BS selects the last user but does not allow it to transmit any information.

Analyzing the mode of operation of the SW-PA scheme, the probability that a user fails to be selected is

$$
P_{F}^{S W-P A}=F_{\gamma_{s_{i}}^{P A}}\left(\gamma_{T}\right) \text {. }
$$

The CDF of $\gamma_{s}^{P A^{*}}$, (the output SNR per time-slot), is as follows

$$
F_{\gamma_{s}^{P A}}^{S W-P A}(x)=\left\{\begin{array}{l}
\beta\left[F_{\gamma_{s}^{P A}}(x)-F_{\gamma_{s}^{P A}}\left(\gamma_{T}\right)\right], x \geq \gamma_{T} \\
P_{n o}^{P A}(x), x<\gamma_{T}
\end{array}\right.
$$

where $\beta=\frac{1-P_{n_{0}}^{P A}}{1-P_{F}^{S W-P A}}$.

The ASE and optimal switching threshold is found in a similar manner to equations (14) and (15) respectively, replacing $F_{\gamma_{s}^{*}}^{S C}(x)$ with $F_{\gamma_{s}^{*}}^{S W-P A}(x)$ and $\mathrm{ASE}_{S C}$ with $\mathrm{ASE}_{S W-P A}$. The average feedback load is given by

$$
{\overline{F_{B}}}^{S W-P A}=\frac{1}{1-P_{F} S W-P A},
$$

and the average delay is given by

$$
\bar{D}^{S W-P A}=\frac{P_{n o}^{P A}}{1-P_{n o}^{P A}} .
$$

\section{NumericAl Results}

The performance of the proposed schemes is illustrated in this section with selected numerical results. These numerical examples are obtained by Monte-Carlo simulations and are confirmed by analytical results. The values of $\overline{\gamma_{s}}=10 \mathrm{~dB}$, $\overline{\gamma_{p}}=0 \mathrm{~dB}, \mathrm{BER}_{0}=10^{-3}$, and $P_{\max }=10 \mathrm{~dB}$ have been fixed for all examples.

Fig. 2 depicts the optimal switching threshold that maximizes the ASE versus the number of users. In general, as the number of users increase, the switching threshold increases. Figure 2 also shows that as the PIC increases, the threshold values increase for both the SW and SW-PA schemes. It is also noted that as the interference threshold increases, the feedback load and delay decrease because more users are able 


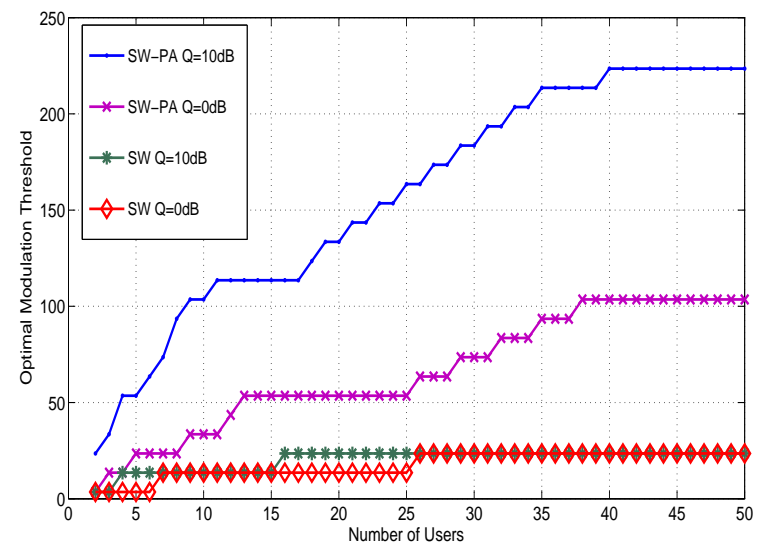

Fig. 2. Optimal switching threshold versus number of users for $Q_{d B}=0$, $\overline{\gamma_{s}}=10 \mathrm{~dB}, \overline{\gamma_{p}}=0 \mathrm{~dB}, \mathrm{BER}_{0}=10^{-3}$, and $P_{\max }=10 \mathrm{~dB}$

to meet the PIC and hence can be candidates for channel access selection.

In Fig. 3, the ASE versus the number of users connected to the BS for all of the schemes are illustrated. It is noticeable that the SC-PA scheme yields the highest ASE. The full power adaptive cases significantly increase the ASE versus the on/off power adaptive cases for both the SC and SW schemes. Comparing the SC scheme to the SW scheme, the ASE for the SC is higher than that of the SW scheme, and similarly for SC-PA and SW-PA.

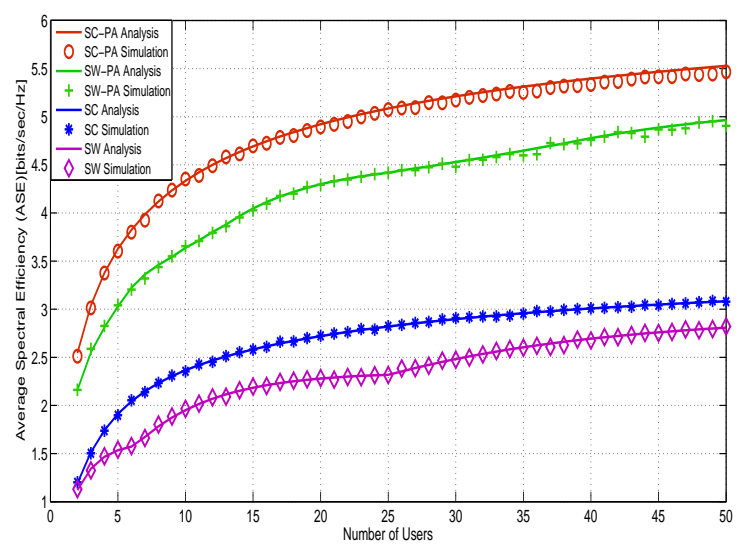

Fig. 3. Average spectral efficiency versus the number of users for $Q_{d B}=0$, $\overline{\gamma_{s}}=10 \mathrm{~dB}, \overline{\gamma_{p}}=0 \mathrm{~dB}, \mathrm{BER}_{0}=10^{-3}$, and $P_{\max }=10 \mathrm{~dB}$

The average feedback load for all the schemes are depicted in Fig. 4. The SC-PA scheme has the highest feedback load due to fact that all users adapt their power to meet the PIC, thus all users must be probed by the BS and therefore the average feedback is linear and equal to the number of users connected to the BS. The SW scheme and the SW-PA scheme both yield less feedback loads than the SC scheme. The jumps in the average feedback load for both the SW and SWPA schemes are due to the changing switching threshold (as depicted in Fig. 2). Comparing the switching thresholds for both the SW and SW-PA schemes in Fig. 2 to the average feedback load for these schemes in Fig. 4, it is noticeable that as the switching threshold jumps from one value to another, the average feedback load exhibits a similar behavior. This is because as the switching threshold increases, it becomes more probable that a user is not able meet the higher switching constraint, and therefore more users must be tested before a successful transmission occurs.

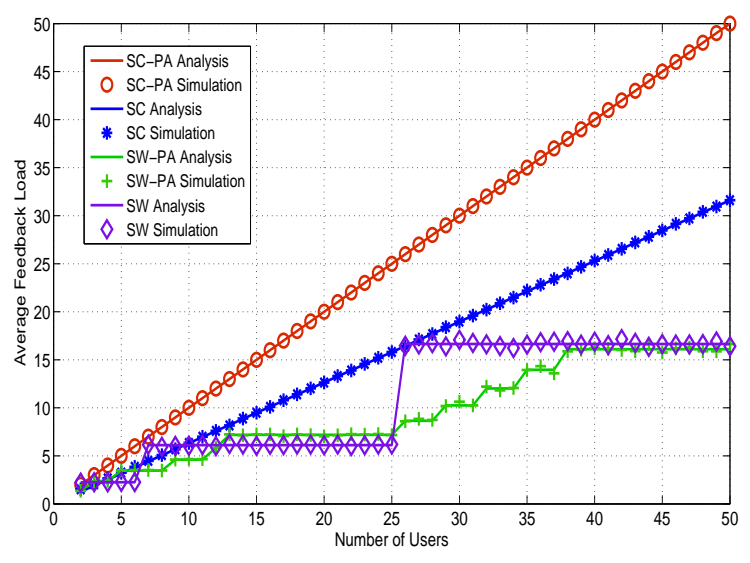

Fig. 4. Average Feedback Load versus the number of users for $Q_{d B}=0$, $\overline{\gamma_{s}}=10 \mathrm{~dB}, \overline{\gamma_{p}}=0 \mathrm{~dB}, \mathrm{BER}_{0}=10^{-3}$, and $P_{\max }=10 \mathrm{~dB}$

In Fig. 5, the average number of no transmissions observed by the BS (average number of delay) is depicted for all the proposed schemes. Since each scheme operates with a different optimal switching threshold, the delay for each scheme is different. For the SW scheme, there are many spikes in the graph. The non-smooth characteristic of the delay is due to the changing value of the optimal switching threshold and the increasing number of users connected to the BS. As the switching threshold increases, it becomes intuitive that the delay will increase since it becomes more probable that users will not be able to satisfy the switching constraint. On the other hand, as the number of users increase, it becomes more likely that an acceptable user is found that meets the constraint. These two opposing factors lead the delay graphs to exhibit such behavior. However, in general, the full power adaptive schemes produce less average delay when compared to the on/off power adaptive schemes.

Figure 6 shows how the average feedback load of each scheme changes as the PIC increases for a total of 10 users connected to the BS. As the PIC constraint increases, more users are able to satisfy the interference constraint and thus for the scan-and-wait schemes, the feedback decreases since it becomes more probable that an acceptable user is found. However, this is the opposite for the selection combining schemes since the number of users that the BS must probe increases as more users meet the PIC. 


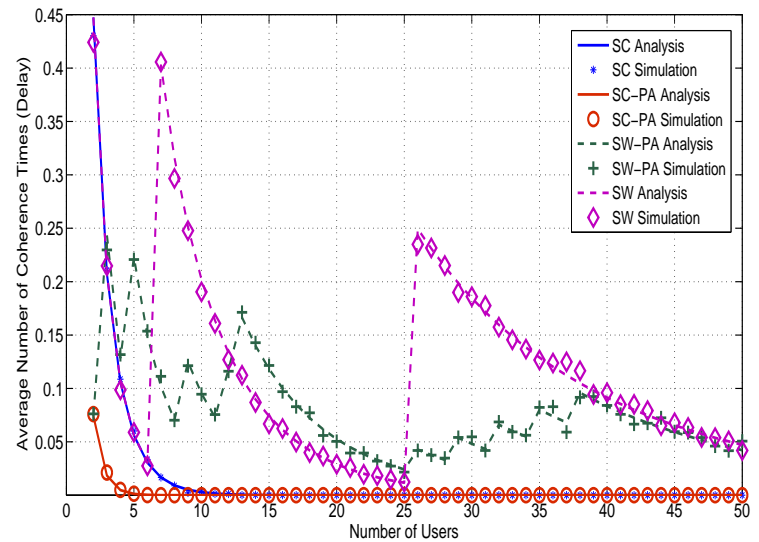

Fig. 5. Average number of coherence times (average delay) versus the number of users for $Q_{d B}=0, \overline{\gamma_{s}}=10 \mathrm{~dB}, \overline{\gamma_{p}}=0 \mathrm{~dB}, \mathrm{BER}_{0}=10^{-3}$, and $P_{\max }=10 \mathrm{~dB}$

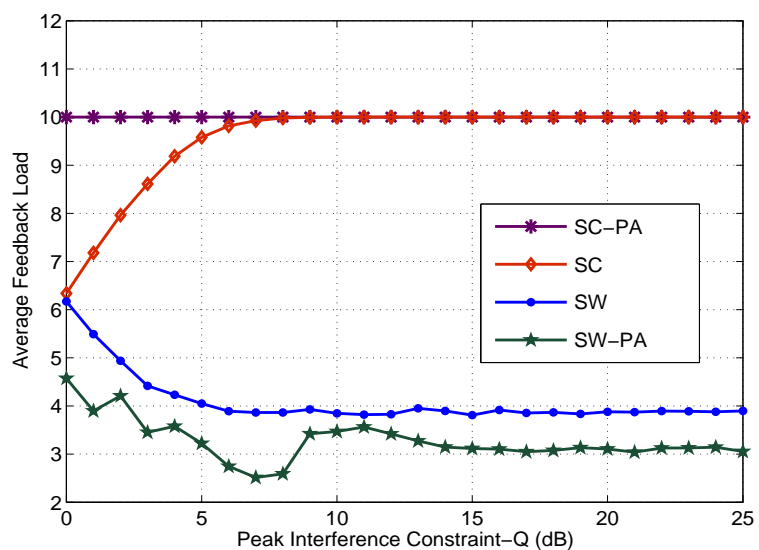

Fig. 6. Average Feedback Load versus the PIC for $\mathrm{K}=10, \overline{\gamma_{s}}=10 \mathrm{~dB}$, $\overline{\gamma_{p}}=0 \mathrm{~dB}, \mathrm{BER}_{0}=10^{-3}$, and $P_{\max }=10 \mathrm{~dB}$

\section{Final Remarks and Further Discussions}

In this study, it is shown that in multiuser systems operating in spectrum sharing networks, scheduling the user based on highest channel quality increases the average spectral efficiency. However, this method is associated with a high feedback load. In an attempt to decrease the feedback load, a switched scheduling scheme is analyzed where a lower average spectral efficiency is observed. Furthermore, transmit power adaptive techniques that ensure that secondary users meet the interference constraint with the primary prove to increase spectral efficiency as well as decrease delay. Future work related to this study includes analyzing the performance of the proposed schemes under the assumption of independent but not necessarily identically distributed Rayleigh fading channels.

\section{ACKNOWLEDGMENT}

This publication was made possible by NPRP grant \# 09341-2-128 and \# 08-152-2-043 from the Qatar National Re- search Fund (a member of Qatar Foundation). The statements made herein are solely the responsibility of the authors.

\section{REFERENCES}

[1] E. FCC, "Docket no 03-222 notice of proposed rule making and order," 2003.

[2] Z. Bouida, M. Abdallah, K. Qaraqe, and M. Alouini, "Spectrally efficient switched transmit diversity for spectrum sharing systems," in Vehicular Technology Conference (VTC Fall), 2011 IEEE. IEEE, 2011, pp. 1-5.

[3] G. Gur, S. Bayhan, and F. Alagoz, "Cognitive femtocell networks: an overlay architecture for localized dynamic spectrum access [dynamic spectrum management]," Wireless Communications, IEEE, vol. 17, no. 4 pp. 62-70, 2010.

[4] B. Holter, M. Alouini, G. Oien, and H. Yang, "Multiuser switched diversity transmission," in Vehicular Technology Conference, 2004. VTC2004Fall. 2004 IEEE 60th, vol. 3. IEEE, 2004, pp. 2038-2043.

[5] H. Yang and M. Alouini, "Performance analysis of multibranch switched diversity systems," Communications, IEEE Transactions on, vol. 51, no. 5, pp. 782-794, 2003.

[6] T. Ban, W. Choi, B. Jung, and D. Sung, "Multi-user diversity in a spectrum sharing system," Wireless Communications, IEEE Transactions on, vol. 8, no. 1, pp. 102-106, 2009.

[7] M. Simon and M. Alouini, Digital communication over fading channels. Wiley-IEEE Press, 2005, vol. 86.

[8] M. Alouini, M. Simon, and H. Yang, "Scan and wait combining (swc): a switch and examine strategy with a performance-delay tradeoff," in Control, Communications and Signal Processing, 2004. First International Symposium on. IEEE, 2004, pp. 153-158.

[9] M. Alouini and A. Goldsmith, "Adaptive modulation over nakagami fading channels," Wireless Personal Communications, vol. 13, no. 1, pp. 119-143, 2000.

[10] K. Hole, H. Holm, and G. Oien, "Adaptive multidimensional coded modulation over flat fading channels," Selected Areas in Communications, IEEE Journal on, vol. 18, no. 7, pp. 1153-1158, 2000.

[11] H. Suraweera, P. Smith, and M. Shafi, "Capacity limits and performance analysis of cognitive radio with imperfect channel knowledge," Vehicular Technology, IEEE Transactions on, vol. 59, no. 4, pp. 1811-1822, 2010. 